\title{
Implantation and Growth of Dendritic Gold Nanostructures on Graphene Derivatives: Electrical Property Tailoring and Raman Enhancement
}

\author{
Kabeer Jasuja and Vikas Berry* \\ Department of Chemical Engineering, 1011 Durland Hall, Kansas State University, Manhattan, Kansas 66506
}

ABSTRACT Interfacing electron-rich metal nanoparticles with graphene derivatives can sensitively regulate the properties of the resultant hybrid with potential applications in metal-doped graphene field-effect transistors (FETs), surface-enhanced Raman spectroscopy, and catalysis. Here, we show that by controlling the rate of diffusion and catalytic reduction of gold ions on graphene oxide (G0), dendritic "snowflake-shaped" gold nanostructures (SFGNs) can be templated on graphene. The structural features of the SFGNs and their interfacing mechanism with $\mathrm{GO}$ were characterized by microscopic analysis and Raman-scattering. We demonstrate that (a) SFGNs grow on G0-surface via diffusion limited aggregation; (b) SFGN's morphology (dendritic to globular), size (diameter of $150-500 \mathrm{~nm}$ and a height of $45-55 \mathrm{~nm}$ ), coverage density, and dispersion stability can be controlled by regulating the chemiophysical forces; (c) SFGNs enhance the Raman signal by 2.5 folds; and (d) SFGNs act as antireduction resist during G0-SFGN's chemical reduction. Further, the SFGNs interfacing with graphene reduces the apparent band gap (from 320 to $173 \mathrm{meV}$ ) and the Schottky barrier height (from 126 to 56 $\mathrm{meV}$ ) of the corresponding FET.

KEYWORDS: graphene - anisotropic gold nanoparticles · hybrid nanostructure - Raman spectra - band-gap - Schottky barrier

*Address correspondence to vberry@ksu.edu.

Received for review May 15, 2009 and accepted July 01, 2009.

Published online July 15, 2009. 10.1021/nn900504v CCC: $\$ 40.75$

๑C 2009 American Chemical Society

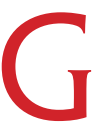
raphene's unique electrical, ${ }^{1,2}$ mechanical $^{3}$ and interfacial ${ }^{4}$ properties, such as (a) high carrier mobility with ballistic transport, ${ }^{1,2}$ (b) mechanically strong carbon-carbon $\mathrm{sp}^{2}$ bonded honeycomb lattice, ${ }^{5}$ (c) confined carrier transport in truly two-dimensional space, ${ }^{6}$ (d) adjustable surface chemistry via chemical $^{7-9}$ or plasma ${ }^{10}$ processes and (e) sensitive response to surface doping, ${ }^{11,12}$ have led to the development of ultrafast electronic devices, ${ }^{1,2,13,14}$ molecular resolution sensors, ${ }^{12}$ biodevices, ${ }^{4}$ polymer composites, ${ }^{15}$ liquid-crystal devices, ${ }^{16}$ electromechanical systems, ${ }^{17}$ and magnetoresistive/quantum-Hall devices. ${ }^{18-20}$ Currently, there is a great interest in functionalization $^{7-9}$ and doping ${ }^{11,12}$ of graphene to manipulate its electrical, ${ }^{7-9,11}$ structural, ${ }^{4}$ and interfacial ${ }^{4}$ properties to enhance its performance. Although, chemical functionalization of graphene results in the formation of scattering sites that reduce its carrier mobility, the thus produced graphene chemical derivatives (GCDs) are appropriate for interfacing with other systems ${ }^{4}$ and for room-temperature electronic applications as they exhibit a suitable band gap/conductivity. Metal functionalization on graphene however needs to be controlled and its effect studied in detail. For this purpose, solution-based gold functionalization of graphene is shown here.

Being structurally distinct from OD molecules and 1D polymers, solvent-dispersed GCDs with quasi-2D structure and tethered chemical groups show novel physiochemical properties. We demonstrate that GCDs function as excellent in-solution substrates for metal nuclei seeding and their subsequent growth into nanodendritic structures. This process, occurring on GO sheets, is governed by diffusion limited transport ${ }^{21}$ of the gold ions with anisotropic lattice incorporation of gold atoms during the seeding growth and is controlled by the masstransfer rates (shown later) (Figure 1a). Here, since the SFGNs are grown on GO, this process is fundamentally different from spherical nanoparticle interfacing ${ }^{7,22-24}$ with graphene, via adsorption, electrochemical, or chemical routes. The highly anisotropic SFGNs, templated on GO sheets $1-4 \mathrm{~nm}$ thick ( $1-5$ layers) and $25-200 \mu \mathrm{m}^{2}$ in area, have predominantly five primary branches with several sharp-edged secondary branches. The SFGNs interfacing on GO leads to (a) increase in conductivity by two folds and (b) 2-fold enhancement of the Raman signals from GO. Further, the GO-SFGN sheets reduced to graphene-SFGN hybrid exhibits an apparent band gap of 164.24 meV and a Schottky barrier height of $38.98 \mathrm{meV}$. We envision 


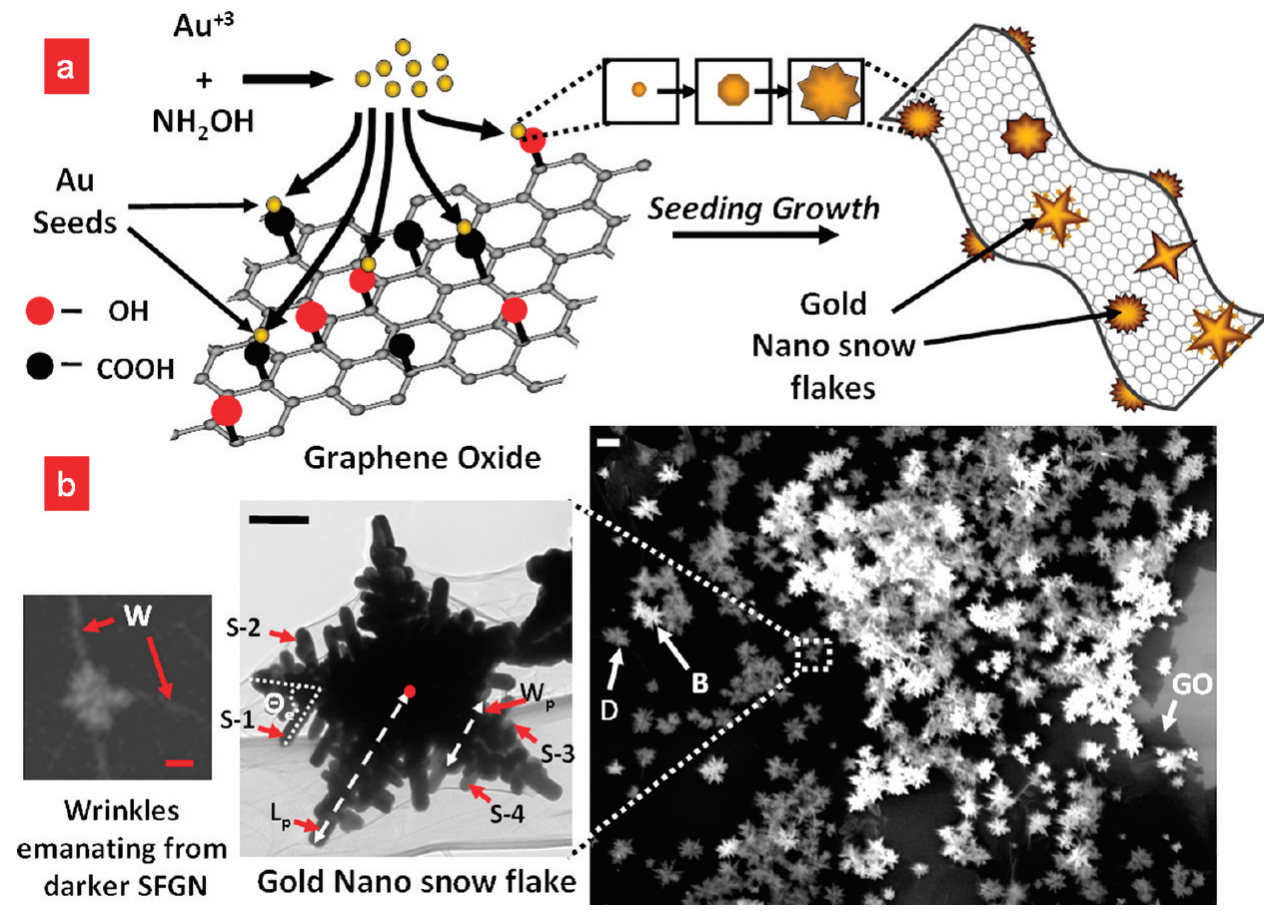

Figure 1. Formation mechanism of snowflake-shaped gold nanostructures on graphene oxide (GO). (a) Interfacing the $-\mathrm{COOH}$ and $-\mathrm{OH}$ groups on $\mathrm{GO}$ sheets with a freshly prepared solution of gold nuclei, formed during hydroxyl-amineassisted reduction of gold salt, results in nuclei attachment and seed-mediated formation of snowflake-shaped gold nanostructures (SFGNs) on the GO surface. (b) Right: FESEM of SFGNs templated on GO lying on silica surface. The SFGNs appearing darker (labeled D) are on the rear surface, while the SFGNs appearing brighter (labeled B) are on the front surface of the immobilized GO sheet (scale bar $=500 \mathrm{~nm}$ ). Center: Higher magnification transmission electron microscopy (TEM) image showing the detailed characteristics and the structural parameters of SFGN exhibiting a dendritic morphology. Scale bar $=100 \mathrm{~nm}$. Left: FESEM image showing wrinkles (labeled W) on GO associated with a darker SFGN. Scale bar $=200 \mathrm{~nm}$.

that the graphene-metallic interfacing will open avenues for next-generation graphene applications in areas including electronics, where the semiconductor properties of graphene could be modulated and integrated with other GCD systems to make graphene logic devices; bioimaging/diagnostic systems, where the surface-enhanced Raman spectroscopy (SERS) from SFGNs could be used for sensing biocomponents and bioprocesses; optoelectronics, where the optical properties of SFGNs could be integrated with the electrical sensitivity of graphene to build solar cells, optical sensors, etc.; nanoheaters, where the IR absorption by anisotropic gold nanostructures could be used to generate thermal energy; and nanocatalysts. ${ }^{24}$

\section{RESULTS AND DISCUSSION}

In this work, Hummers method ${ }^{8,25}$ is used to prepare graphene oxide (GO), having oxy-functional groups such as carboxyl $(-\mathrm{COOH})$, hydroxyl $(-\mathrm{OH})$, and epoxy groups ${ }^{4}$ on its surface. In $\mathrm{GO}$ suspension, these oxy-functional groups are leveraged to stabilize gold nuclei, synthesized in situ by hydroxyl-amine assisted reduction of gold salt. The gold nuclei are subsequently grown via the seeding growth mechanism (Figure 1a) to synthesize the GO-SFGN hybrid by mixing $1.3 \mu \mathrm{L}$ of hydroxylamine $\left(\mathrm{NH}_{2} \mathrm{OH}, 50 \% \mathrm{w} / \mathrm{v}\right)$ to $50 \mathrm{~mL}$ of $0.275 \mathrm{mM}$ gold salt $\left(\mathrm{HAuCl}_{4} .3 \mathrm{H}_{2} \mathrm{O}\right)$ followed by an addition of $100 \mu \mathrm{L}$ of graphene oxide (GO) suspension (80
$\mathrm{mM}$ carboxylic acid, quantified by titration). After constant agitation at room temperature for $1 \mathrm{~h}$, the resultant GO-SFGN hybrid sheets are immobilized on an amine-silanized silica substrate (see Supporting Information, Figure S1). Field emission scanning electron microscopy (FESEM) (Figure 1b, right) indicated excellent templating of SFGNs on GO. The SFGNs appearing darker under FESEM (labeled as " $\mathrm{D}$ ") are probably a result of their attachment on the rear GO surface (facing silica), which shields the SFGNs giving lesser average surface-electron-density. The brighter SFGNs (labeled as " $\mathrm{B}$ ") are templated on the exposed GO-surface. This is expected since SFGNs will nucleate on both sides of the GO-sheets exposed to the solution. This is further evidenced by the wrinkles emanating on the GO sheets from darker SFGNs (beneath the GO sheet) (Figure 1b left, see Supporting Information Figure S2), which are expected to raise, stretch, and therefore wrinkle the GOsheets around them.

Figure 2a shows FESEM micrographs of SFGNs with five and six primary branches $\left(N_{\mathrm{p}}\right)$ and several secondary or side branches. The presence of these secondary branches makes the SFGNs fundamentally different from multipod and star-shaped gold nanostructures. ${ }^{26-29}$ TEM (Figure 1b, center) of an SFGN with five primary branches $\left(N_{p}=5\right)$, shows an average primary branch length $\left(L_{p}\right)$ of $\sim 260 \mathrm{~nm}$ and an average width $\left(W_{\mathrm{p}}\right)$ (the maximum thickness of the pri- 


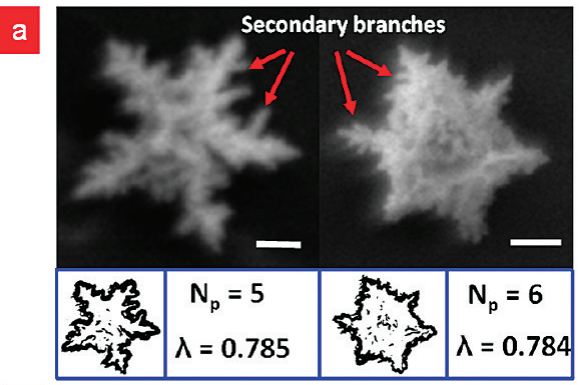

b
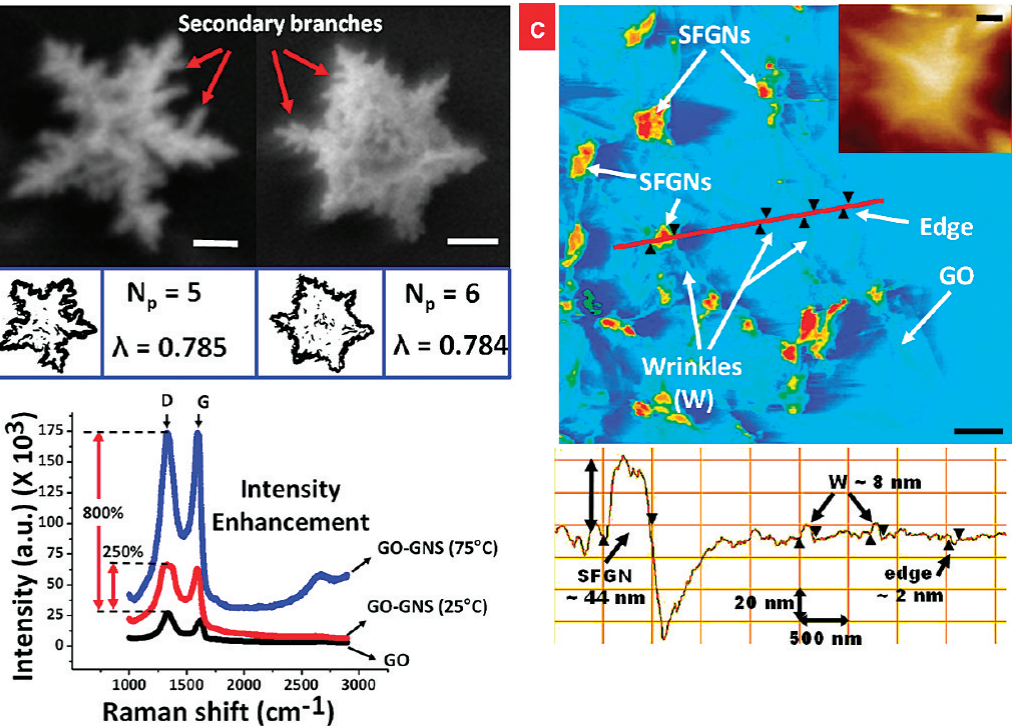

Figure 2. "Snowflake"-shaped dendritic morphology of the gold nanostructures and AFM scan of GO-SFGN hybrid. (a) FESEM images of individual SFGNs with 5 and 6 primary branches $\left(N_{\mathrm{p}}\right)$, respectively, showing a distinct secondary branching (scale bar $=100 \mathrm{~nm}$ ). Bottom insets show binary edge-resolved images for these SFGNs used for determining the circularity parameter $\left(\lambda=4 \pi^{*}\right.$ area/perimeter $\left.{ }^{2}\right)$. Values smaller than unity indicate a high degree of corrugated edges. (b) Raman Spectra for GO-SFGN and GO showing the presence of SFGNs on GO enhances the intensity of D and G bands by $\sim 250 \%$ and $\sim 800 \%$ suggesting chemical enhancement, and hence a chemical-bond formation between SFGNs and GO, (c) atomic force microscopy scan of GO sheets templated with SFGNs with $\sim 44$ $\mathrm{nm}$ height as shown in the bottom line scan (scale bar $=500 \mathrm{~nm}$ ). The height of a characteristic wrinkle $(W)$ is $\sim \mathbf{8} \mathbf{n m}$. Inset shows AFM image for an individual SFGN (scale bar $=$ $100 \mathrm{~nm})$.

mary branch at the nodal point) of $\sim 120 \mathrm{~nm}$. Each primary branch backbone structure emanates parallel secondary or side branches with the same angles of emergence $\left(\Theta_{\mathrm{e}}\right)$ (the angle between a side branch and its primary branch backbone) which vary from $\sim 35^{\circ}$ to $\sim 90^{\circ}$ from branch to branch. $\Theta_{\mathrm{e}}$ for side branches labeled S-1 and S-2 is $\sim 60^{\circ}$ while that for the side branches labeled S-3 and S- 4 is $\sim 80^{\circ}$ (Figure $1 \mathrm{~b}$, center). This indicates that the secondary-branch's growthdirection is influenced by the crystal-lattice of their common primary branch rather than a random nucleation process. The longer secondary branches closer to the center than those near the tip, indicate that nearcenter secondary branches are exposed to the growth mixture for a longer time, implying a progressive growth of the primary branches from nuclei. Also, as shown later, a more pronounced secondary branching and instances of ternary branches in SFGNs can be obtained by increasing the concentration of gold ions in solution. Further characterization for more SFGNs is summarized in Table S1, Supporting Information. Anisotropy as evaluated by circularity parameter $(\lambda=$ $4 \pi^{*}$ area/perimeter ${ }^{2}$ ), as expected showed $\lambda<1$, characteristic for branched structures (Figure $2 \mathrm{a}$, bottom insets).

The effect of interaction of SFGNs with the GOsurface was probed by Raman scattering signal analysis for bare GO and GO-SFGN sheets (Figure 2b). Raman spectrum for a GO sheet exhibits the regular two peaks, corresponding to the D-band line $\left(\sim 1340 \mathrm{~cm}^{-1}\right)$ and the G-band line $(\sim 1590$ $\left.\mathrm{cm}^{-1}\right)$. SFGN-interfacing on GO enhanced the intensity of these bands by $>250 \%$ (Figure 2b). Surface enhancement of Raman signals can be via electromagnetic enhancement (excitation of localized surface plasmons involving physical interaction) or chemical enhancement (formation of charge-transfer complexes involving chemical interaction) with enhancement factors of $\sim 10^{12}$ and $\sim 10$ to 100 , respectively. ${ }^{30,31}$ The low enhancement factor for GO-SFGN hybrid indicates the presence of a chemical interaction or bond between SFGNs and GO. The Raman signal enhancement is similar to the SERS effects previously reported in metal nanoparticle composites of carbon nanotubes. ${ }^{32}$ As shown later, a Raman enhancement factor of $\sim 800 \%$ is achieved by increasing the density of gold nanostructures on GO sheets by changing the synthesis temperature to $\sim 75^{\circ} \mathrm{C}$ (Figure 2b).

Atomic force microscopy (AFM) scans on the GO-SFGN hybrids (Figure 2c) shows three regions: (i) large peaks corresponding to the SFGNs, (ii) small peaks corresponding to the wrinkles on $\mathrm{GO}$, and (iii) small dips corresponding to the edge of the $\mathrm{GO}$ sheets (shown in the bottom panel). The height of the SFGN structure was $\sim 44 \mathrm{~nm}$ and varies between $\sim 45$ and $55 \mathrm{~nm}$ (see Supporting Information, Figure S3), while the GO wrinkles were $\sim 8$ $\mathrm{nm}$ high. AFM-tip curvature and low contrast of the thick SFGN structures hid the details of the primary and secondary branches of individual SFGN (Figure 2c, top inset). Further, the SFGNs from which wrinkles are emanating are expected to be underneath the GO sheet, as shown in Figure $1 \mathrm{~b}$.

The lower height of the SFGNs than their width and their flat surface being level with the GO sheets suggest that the SFGNs growth direction is more favored laterally than vertically to the GO surface. This indicates that the GO sheets functions as an in-solution substrate for particle growth. However, more studies are required to confirm this. In the absence of $\mathrm{GO}$, the seeding solution $\left(\mathrm{NH}_{2} \mathrm{OH}\right.$ and gold salt) results in the formation of large irregular aggregates, which eventually settle down. This further signifies the role of GO sheets as stabilizing agents for controlled growth of SFGNs. SFGN-GO solutions were stable for $>20$ days. Absorption spectra for a freshly prepared solution of SFGN-GO exhibits a broad peak at $\sim 580 \mathrm{~nm}$ (Figure 3) suggesting the presence of colloidal aggregates in solution as well. Further, the SFGNs grown on GO for 20 
days were higher in number but with a size and shape distribution similar to that for freshly prepared SFGNs.

The dendritic shape of SFGNs resemble (i) the morphology of naturally occurring snowflakes, where the dendritic structures are generated from water molecules condensing via diffusion-limited mechanism on supercooled water droplets (snow nuclei) and (ii) the morphologies proposed for dendritic growth patterns due to molecular anisotropy. ${ }^{33}$ These structures follow an adapted diffusion limited growth pattern model, where particles moving in random walk trajectories stick on a lattice containing a seed particle anisotropically (due to microscopic irregularities on the surface) with the seed tips growing preferentially. A modified two-step model is proposed here for the mechanism of formation of SFGNs. The first step involves the formation of gold nuclei on $\mathrm{GO}$ via $\mathrm{NH}_{2} \mathrm{OH}$-assisted reduction of gold salt and their stabilization via attachment with the negatively charged $-\mathrm{COO}^{-}$and $-\mathrm{OH}^{-}$groups on $\mathrm{GO}$. We confirmed this step by growing gold nuclei on $\mathrm{GO}$ by sodium borohydride reduction of gold salt in the presence of sodium citrate as shown in Figure 4a, bottom inset (and Supporting Information, Figure S4).

Since the SFGN formation is an aqueous phase process and unmodified graphene cannot be dispersed in water without surface modification or surfactants, achieving the SFGN formation on graphene (without the $-\mathrm{COO}^{-}$ and $-\mathrm{OH}^{-}$groups) is challenging. Further, it is also difficult to control the density of $-\mathrm{COO}^{-} / \mathrm{OH}^{-}$groups on $\mathrm{GO}$, since during the exfoliation process, the degree of oxidation of sheets is expected to be higher for sheets exfoliating early and thus exposed to the oxidizing media for longer time. Therefore, in a single batch, different GO sheets will have different oxy-group densities, making it challenging to study the effect of oxygroups on SFGN deposition characteristics.

The second step involves seeding growth of the gold nucleus induced by hydroxyl-amine assisted $\mathrm{Au}$ (III) reduction, catalyzed by the gold surface ${ }^{34}$ of the nuclei. The dendritic morphology of these gold nanostructures (GNs) is attributed to the diffusion limited kinetics during the seeding growth. The seeding growth of gold nuclei on GO has further two elementary processes (Figure 4a): (a) external diffusion of Au (III) ions from bulk solution to the nuclei and; (b) particle incorporation of the Au atoms into the crystal structure by hydroxylamine-induced catalysis. The heat of SFGNs formation at nanoscale is removed by the surrounding water. The net resistance to SFGNs growth is thus a combination of resistances from these elemental steps:

$$
k_{\mathrm{F}}^{-1}={k_{\mathrm{D}}}^{-1}+{k_{\mathrm{G}}}^{-1}
$$

where $k_{\mathrm{F}}$ is the rate-constant for SFGN formation, $k_{\mathrm{D}}$ is the rate-constant for diffusion of $\mathrm{Au}$ ions from the bulk solution on to the GO surface, and $k_{\mathrm{G}}$ is the rateconstant of $\mathrm{Au}$ incorporation in the growing SFGN

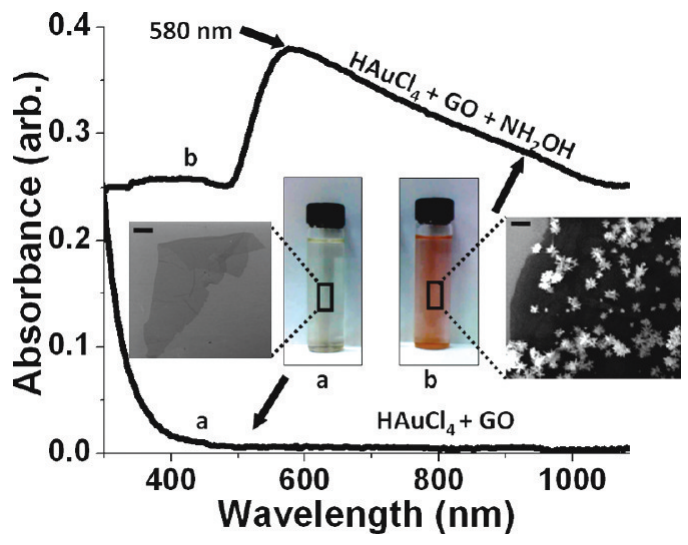

Figure 3. Absorption spectra of SFGNs templated on graphene-oxide: (a) spectra of gold salt and GO display no significant peak in the visible region; (b) spectra of a freshly prepared solution of GO, gold salt, and hydroxyl-amine display an absorption peak at $\sim \mathbf{5 8 0} \mathbf{n m}$. Higher magnification FESEM micrographs (insets) for a bare GO sheet and of a GO sheet templated with SFGNs immobilized on silica substrates are shown. Scale bar equals $1 \mu \mathrm{m}$ for the right inset and $5 \mu \mathrm{m}$ for the left inset.

structure. Since particle incorporation step is catalytic, $k_{\mathrm{G}}$ is expected to be high, making external diffusion the rate determining step. This is further evidenced by the SFGN's structural dependence on the temperature of synthesis (Figure 4b) as studied by comparing the FESEM images of nanostructures synthesized at 4, 25, and $75^{\circ} \mathrm{C}$. The SFGNs are produced only at $25^{\circ} \mathrm{C}$, while the dendritic morphologies are not produced at low (4 ${ }^{\circ} \mathrm{C}$ ) or at high $\left(75^{\circ} \mathrm{C}\right)$ temperatures. The size and density of the nanoparticles were found to increase with an increase in the temperature. The surface density of GNs was quantified by evaluating the surface coverage index $\eta$ ( $\eta=$ fraction of GO surface covered by gold nanostructures). $\eta$ for low, moderate, and high temperatures were calculated to be $0.06,0.24$, and 0.41 , respectively, as shown in Figure $4 \mathrm{~b}$ inset.

Since the diffusive resistance, $k_{\mathrm{D}}{ }^{-1} \propto T^{-1.5}$, and particle incorporation resistance, ${k_{\mathrm{G}}}^{-1} \propto \exp \left(E_{\mathrm{A}} /(R T)\right)$ is higher for low temperature, smaller particles are formed with low density. Here, the particle-incorporation resistance is expected to be higher than that due to diffusion. At moderate temperature of $25^{\circ} \mathrm{C}$, the diffusive resistance is thought to increase more than the particleincorporation resistance leading to SFGNs formation. The anisotropic particle growth on the SFGN surface is a result of the difference in the surface chemical potential $(\mu)$ induced by small deformations. Irregular surfaces with high $\mu$ grow more preferentially than blunt surfaces with low $\mu[\mu(R=\infty)<\mu(R=$ constant $)$ (Mullins-Sekereka instability). ${ }^{35,36}$ The average rate of gold influx for the formation of an SFGN $\left(N_{p}=5\right)$ is estimated to be $\sim 0.1346 \mathrm{~g} \mathrm{~cm}^{-2} \mathrm{~h}^{-1}$ at $25^{\circ} \mathrm{C}$ (see Supporting Information Figure S5). At higher temperature $\left(75^{\circ} \mathrm{C}\right)$, the mass-transfer rates and the chemical potentials increase leading to a fast and thus unordered cluster formation with high surface coverage densities (Fig- 

brids synthesized at these three temperatures.

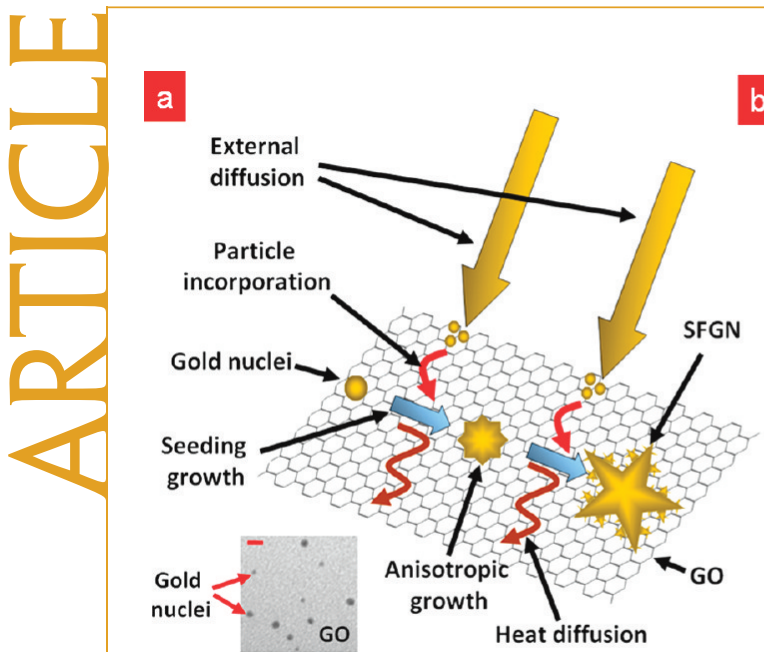

Figure 4. Growth mechanism of SFGNs on GO sheets and their structural dependence on the synthesis temperature. (a) Schematic showing the elementary steps involved in the seeding growth of SFGNs on GO template. Au ions diffuse from the bulk to the GO sheet where they are catalytically reduced and incorporated in the growing Au nuclei. Bottom inset shows seed particles on GO that were prepared by sodium borohydride-assisted reduction of gold salt in the presence of sodium citrate and GO. Scale bar $=10 \mathrm{~nm}$. (b) The morphology and density of the synthesized GNs sensitively depends on the reaction temperature. GNs synthesized at room temperature $\left(25^{\circ} \mathrm{C}\right)$ exhibit dendritic "snowflake" morphology with a high coverage on GO. At low temperature $\left(4^{\circ} \mathrm{C}\right)$, the $\mathrm{GNs}$ assume a spherical morphology with less coverage, and at higher temperatures $\left(75^{\circ} \mathrm{C}\right)$, GNs exhibit a random cluster formation with very dense coverage. Scale bars $=500 \mathrm{~nm}$. Inset shows the variation of surface coverage index for GO-gold hy-

ure 5c) (see also Supporting Information, Figure S6). The high selectivity of deposition further confirms the role of GO's functional groups in gold nucleation. The

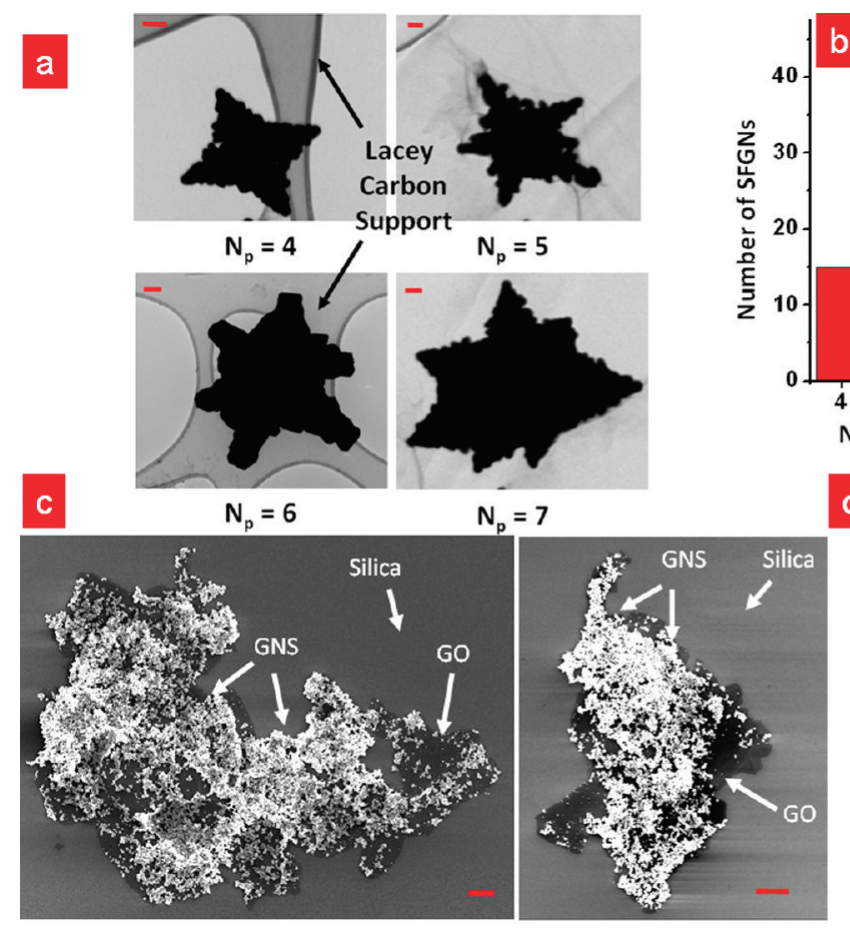

functionalized silica substrate placed in the seeding solution did not form the SFGNs at $25^{\circ} \mathrm{C}$, indicating the importance of segregation of solution by GO.

GO-Au nanostructure hybrid synthesized at $75^{\circ} \mathrm{C}$ was compared with the GO-SFGN hybrid synthesized at $25^{\circ} \mathrm{C}$ for its SERS signals. While the enhancement factors for GO-Au nanostructures synthesized at $25^{\circ} \mathrm{C}$ was $\sim 250 \%$, the GO-Au nanostructures synthesized at $75^{\circ} \mathrm{C}$ exhibited a higher enhancement factor of $\sim 800 \%$ (Figure $2 \mathrm{~b}$ and Supporting Information, Figure 57). The observed higher enhancement factor is expected since more metal is deposited at a higher temperature. Further, it was found that by increasing the concentration of gold ions in the solution, a more pronounced growth of secondary branches can be achieved, which in some cases emanate ternary branches (see Supporting Information, Figure S8). The pronounced secondary growth can be explained by the higher concentration of gold ions that are available for particle incorporation during seeding growth. Interestingly, carboxyl/hydroxyl-
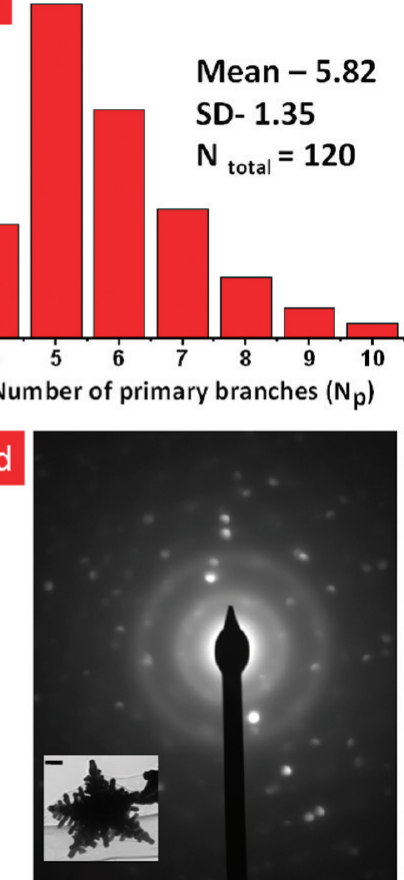

Figure 5. Structural variation in the morphology of SFGNs and diffraction pattern studies. (a) TEM images of SFGNs with 4, 5,6 , and 7 primary branches. Scale bar $=100 \mathrm{~nm}$. (b) Bar graph showing the distribution of primary branches in the SFGNs synthesized at $25^{\circ} \mathrm{C}$. The analysis is for a total of 120 SFGNs deposited on GO. (c) FESEM images for gold-nanostructuretemplated GO sheets that were synthesized at $70^{\circ} \mathrm{C}$ showing high surface coverage densities and excellent selectivity of gold on GO. Scale bar $=5 \mu \mathrm{m}$. (d) Selected area electron diffraction (SAED) pattern for an SFGN with $N_{\mathrm{p}}=5$ (inset) shows that SFGNs have several defects and multiple crystal domains. Scale bar $=100 \mathrm{~nm}$. 

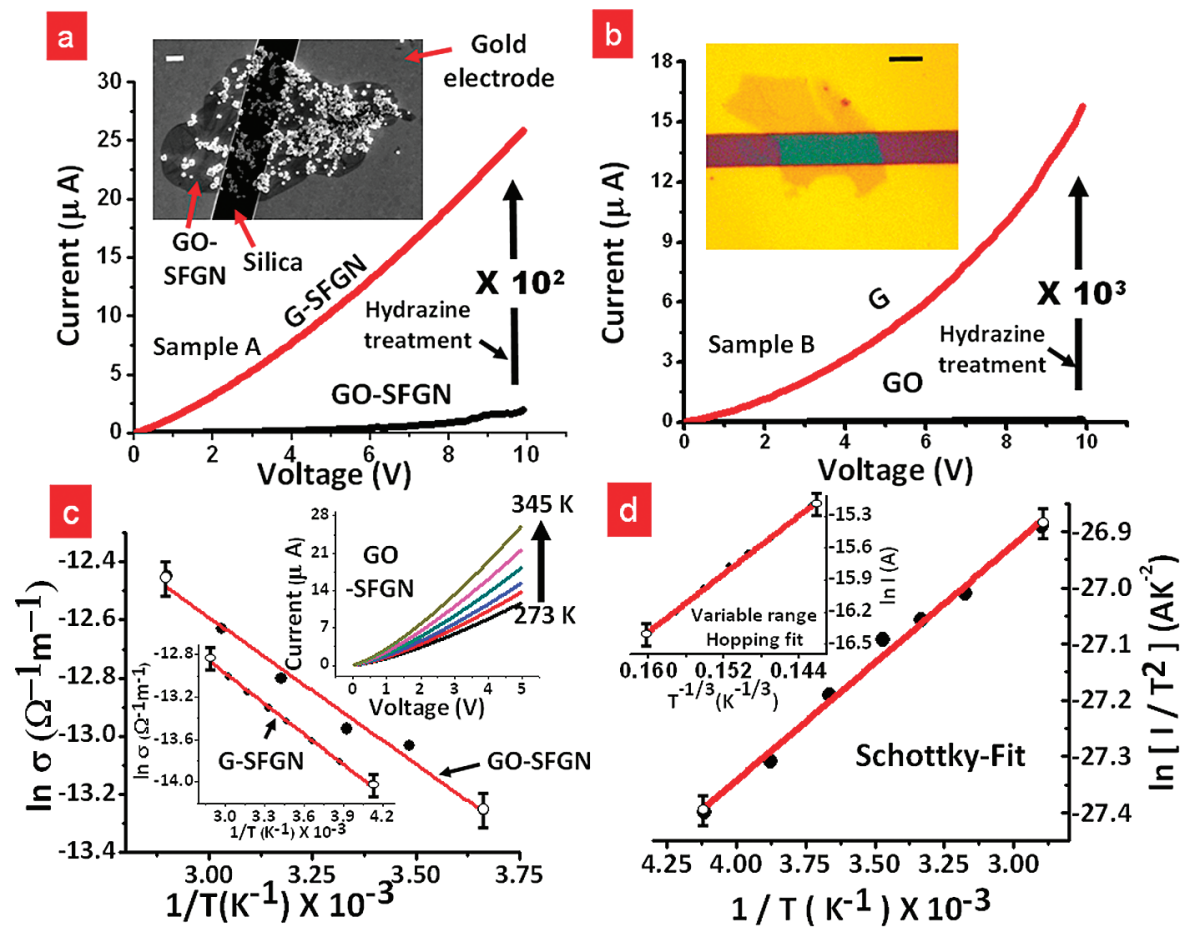

Figure 6. Electrical properties of graphene-templated with SFGNs. (a) The conductivity of a GO-SFGN sheet $\left(9.49 \times 10^{-2}\right.$ $\mu \mathrm{S}$ ) increases $\sim 10^{2}$-fold after reduction with hydrazine. Inset shows a GO sheet templated with SFGNs incorporated between electrodes. Scale bar $=2 \mu \mathrm{m}$. (b) Conductivity of a bare GO sheet increases $10^{3}$ folds after hydrazine reduction. Top inset: Optical micrograph of a bare GO sheet between gold electrodes. Scale bar $=5 \mu \mathrm{m}$. (c) GO-SFGN hybrid exhibits a semiconducting behavior showing an exponential dependence of conductivity on temperature $\left(\sigma \alpha \exp \left(-E_{g} /\left(2 k_{B} T\right)\right)\right.$. Bottom left inset shows a similar temperature-response for a G-SFGN device. Top inset shows the $I-V$ response for a GO-SFGN device over a temperature range of 273-345 K showing a gradual increase in conductivity. (d) Schottky fit for a G-SFGN device from which the Schottky barrier height is calculated to be $\sim 39 \mathrm{meV}$. Top inset shows the variable range hopping fit for G-SFGN, which describes the data equally well.

Figure 5a,b shows the TEMs for SFGNs (synthesized at $25^{\circ} \mathrm{C}$ ) with $N_{\mathrm{p}}=4,5,6$, and 7 and the distribution of $N_{\mathrm{p}}$ for SFGNs synthesized at $25^{\circ} \mathrm{C}$, respectively. While, $N_{\mathrm{p}}$ ranges from 4 to 12 , several SFGNs $(\sim 49.2 \%)$ have $N_{\mathrm{p}}=5$ or 6 , with a relatively small number of SFGNs having $N_{p} \geq 8$ ( $\left.\sim 11.8 \%\right)$. The selected area electron diffraction (SAED) pattern (Figure $5 \mathrm{~d}$ ) of an SFGN with $N_{\mathrm{p}}=5$ (Figure $5 \mathrm{~d}$-inset) exhibited a mixed diffraction pattern, suggesting the presence of defects and multiple crystal domains in the SFGNs. Furthermore, the crystal defects were lesser for SFGNs with incompletely formed secondary branches (Supporting Information Figure S9). The similar angle of emergence of the secondary branches suggests that similar crystal defects cause the secondary branching on a primary branch.

Electrical measurements on an SFGN-GO sheet and a bare-GO sheet immobilized on separate silica substrates with predeposited electrodes were conducted by immobilizing the sheet from solution on amine-functionalized silica substrates with $300 \mathrm{~nm}$ thick silica and gold electrodes $5 \mu \mathrm{m}$ apart (insets, Figure $6 a, b)$. The conductivity of GO-SFGN sheet was an order of magnitude higher than that for $\mathrm{GO}$, attributed to the formation of low-resistance, gold-doped islands on GO. Next, the GO regions in both GO-SFGN and bare-GO devices were chemically reduced to graphene (G) by treatment with hydrazine vapors ${ }^{8}\left(R_{\mathrm{G}} \ll R_{\mathrm{GO}}\right)$. Af- ter $\mathrm{GO}-\mathrm{SFGN}$ to $\mathrm{G}-\mathrm{SFGN}$ reduction (Figure $6 \mathrm{a}$ ), the conductivity increased by 2 orders of magnitude; while for $\mathrm{GO}$ to $\mathrm{G}$ reduction (Figure $6 \mathrm{~b}$ ) it increased by 3 orders of magnitude. The lesser conductivity-change observed in GO-SFGN (100-fold) versus GO (1000-fold) post reduction is attributed to the presence of SFGNs, which shield the underlying GO against hydrazine treatment, resulting in a net smaller region of $\mathrm{GO}$ being reduced to $\mathrm{G}\left(R_{\mathrm{G}}<R_{\mathrm{SFGN} / \mathrm{GO}}\right)$. The $\mathrm{SFGN}$ shielding of $\mathrm{GO}$ against hydrazine treatment is further verified by comparing the band gap $\left(E_{\mathrm{g}}\right)$ values of GO-SFGN and G-SFGN hybrids obtained by measuring the temperature dependence of their conductivities. Figure $6 c$ shows an exponential temperature-dependence $[\sigma \alpha$ $\left.\exp \left(-E_{\mathrm{g}} /\left(2 k_{\mathrm{B}} T\right)\right)\right]$ for the conductivity of a GO-SFGN and $\mathrm{GO}$ devices, from the slope of which their apparent band gap values are calculated to be $E_{\mathrm{g} / \mathrm{GO}-\mathrm{SFGN}}=$ $173.85 \pm 0.01 \mathrm{meV}$ and $E_{\mathrm{g} / \mathrm{GO}}=320.05 \pm 0.01 \mathrm{meV}$, respectively (Supporting Information, Figure S10). The decreased band gap in GO-SFGN explains its higher conductivity than $\mathrm{GO}$ as observed earlier. The conductivity of a G-SFGN device exhibits a similar temperature dependence (Figure $6 c$, bottom left inset) with a band gap of $E_{\mathrm{g} / \mathrm{G}-\mathrm{SFGN}}=164.24 \pm 0.003 \mathrm{meV}$ comparable to $E_{\mathrm{g} / \mathrm{G}}$ $=156.64 \pm 0.01 \mathrm{meV}$ for graphene (chemically reduced GO) (Supporting Information, Figure S10). The band gap of G-SFGN and G being nonzero can be attributed 
to the partial reduction of GO on the unexposed sidefacing silica surface ${ }^{37}$ and defects. The higher band-gap value for $\mathrm{G}-\mathrm{SFGN}$ as compared to $\mathrm{G}\left(E_{\mathrm{g} / \mathrm{G}}<E_{\mathrm{g} / \mathrm{G}-\mathrm{SFGN}}\right)$ results from the presence of unreduced $G O$ regions in the G-SFGN hybrid making G-SFGN less metallic than $G$. Therefore it can be inferred that the expected band-gap values for $\mathrm{GO}-\mathrm{Au}$ hybrids synthesized at $75^{\circ} \mathrm{C}$ should be higher due to the high density of gold nanostructures, which shield larger $\mathrm{GO}$ regions against reduction. Further, the G-SFGN and GO-SFGN hybrids can be modeled as several back-to-back semiconductor-metal interfaces (Schottky contacts), the current through which at a forward bias $V$ is described by

$$
I=A^{*} a T^{2} \exp \left(\frac{-q\left(\phi_{\mathrm{B}}-V\right)}{k_{\mathrm{B}} T}\right)
$$

where $A^{*}$ is the modified Richardson constant, $a$ is the cross sectional area, $\varphi_{B}$ is the Schottky barrier height, $k_{B}$ is the Boltzmann constant and $T$ is the absolute temperature. Figure $6 \mathrm{~d}$ shows the plot of $\ln \left(/ / T^{2}\right)$ versus $1 / T$ data for a G-SFGN device exhibiting a linear dependence which is consistent with the eq 2. The Schottky barrier height (SBH) for G-SFGN hybrid as calculated from the slope of this curve (see Supporting Information) is $38.98 \pm 0.06 \mathrm{meV}$. Similarly the $\mathrm{SBHs}$ for $\mathrm{GO}$, GO-SFGN, and G (chemically reduced GO) were calculated to be $125.98 \pm 0.27,52.63 \pm 0.20$, and $37.36 \pm$ $0.08 \mathrm{meV}$, respectively, indicating that the SBH in GO decreases as it forms a composite with the SFGNs. This holds similarity to the observed decrease of SBH in silicon carbide-Ti Schottky contacts after deposition of gold nanoparticles. ${ }^{38}$ Further, the experimental data for the $I-V$ scans of GO, G, GO-SFGN, and G-SFGN also fits the variable range hopping (VRH) mechanism ( $\ln / \alpha$ $\left.T^{-1 / 3}\right)$, which involves consecutive inelastic tunneling. Figure $6 \mathrm{~d}$ inset shows the VRH fit for a G-SFGN device (VRH fits for GO, G, and GO-SFGN are shown in Figure S11, Supporting Information). However, Schottky limited charge transport should be the preferred mode of charge transport as evidenced by a nonlinearity in the $I-V$ curves shown in Figure $6 a, b$. VRH in graphene has been shown to produce linear $I-V .^{39}$ Further, the formation of Schottky contacts at the interface between metal electrode and graphene sheet is expected since the graphene sheet is contacted via a bottom goldelectrode configuration, which introduces significant Schottky barrier at the electrode edges producing a nonlinear $I-V$ behavior. ${ }^{39}$ However, more experiments are required to analyze the individual roles of $\mathrm{VRH}$ and Schottky limited charge transport in graphene-gold devices.

\section{CONCLUSION}

We have demonstrated the electrical, structural, interfacial, and dendritic properties of controlled interfacing of graphene with gold nanostructures, its electrical properties, and Raman signal can be sensitively controlled. With continued interest in graphene technology, its metal-interfacing will be an important process both for its incorporation into other systems and for controlling its electrical properties by doping. Here, we leverage the molecular-functionality of $\mathrm{GO}$ in dispersion to grow and stabilize gold nanostructures with morphology controlled by manipulating the chemical and physical forces (diffusion and surface-potential). A 2-fold Raman enhancement and a control on the band gap and Schottky barrier was demonstrated. Since, metal nanostructures can sensitively tailor graphene's electrical and Raman properties, we envision that the research community will leverage the process shown here to build novel graphene applications in the areas of nanoelectronics, sensors, bioimaging, electro-optics, catalysis, logic-devices, etc. The study will also motivate further developments in this field by incorporating graphene's fermionic-confinement, spintronics effects, magnetic effects, and carrier collimation to develop next-generation conjugated devices with new functionalities.

\section{EXPERIMENTAL METHODS}

Synthesis of G0-SFGN Hybrids. The GO-SFGN hybrids were synthesized by mixing $1.3 \mu \mathrm{L}$ of $(50 \% \mathrm{w} / \mathrm{v})$ hydroxyl-amine (Sigma Aldrich) to $50 \mathrm{~mL}$ of $0.275 \mathrm{mM}$ gold chloride trihydrate (Fisher Scientific) followed by an addition of $100 \mu \mathrm{L}$ of graphene-oxide suspension ( $80 \mathrm{mM}$ carboxylic acid, quantified by titration). The mixture is kept under constant agitation (100 rpm) at room temperature for $1 \mathrm{~h}$ which results in the formation of SFGNs on GO sheets that can be immobilized on an amine-functionalized silica substrate.

Preparation of Graphene-0xide. To prepare the GO sheets, $5 \mathrm{~g}$ of Mesh 7-graphite flakes were mixed with $33 \mathrm{~mL}$ of $68 \%$ nitric acid $+200 \mathrm{~mL}$ of $96 \%$ sulfuric acid and stirred continuously for 40 min in an ice bath; 30 grams of potassium permanganate was then added into the solution, while the temperature was slowly increased to $40^{\circ} \mathrm{C}$, and kept at $40^{\circ} \mathrm{C}$ for $30 \mathrm{~min}$. Subsequently, the excess potassium permanganate was removed by treatment with $10 \%$ hydrogen peroxide. Finally, the GO sheets were ob- tained by centrifuging this solution at $15000 \mathrm{rpm}$ for $30 \mathrm{~min}$ followed by repeated washing with DI water. The sample was then dialyzed (MWCO 2000D) for $24 \mathrm{~h}$ and the subsequently stored as a suspension in DI water at room temperature. All the chemicals used in this process were obtained from Fisher Scientific.

Immobilizing the G0-SFGN Hybrids on Silica Surface. The GO sheets functionalized with metal nanostructures were immobilized via electrostatic interactions on a heavily doped n-type silicon substrate with a $300 \mathrm{~nm}$ thick thermally grown silica layer. Briefly the substrate is first exposed to oxygen plasma (100 W, 2 mbar, 2 min) followed by treatment with 3-amino propyl triethoxy silane(Gelest) that makes the surface positively charged. This amine-functionalized substrate is then baked for $4 \mathrm{~min}$ at 120 ${ }^{\circ} \mathrm{C}$ and then momentarily exposed ( $\sim 2 \mathrm{~min}$ ) to the aqueous dispersion of GO, hydroxyl amine, and gold salt. The substrate is then thoroughly washed with deionized (DI) water that facilitates electrostatic and selective deposition of GO-SFGN hybrids on silica. 
TEM and SAED. TEM images and SAED patterns were obtained with a Philips CM 100 transmission electron microscope operated at $100 \mathrm{kV}$. The GO-SFGN hybrids were deposited from solution on to 300 mesh size copper TEM specimen grids (Electron Microscopy Sciences) having a carbon support film.

FESEM and EDS. FESEM Images and EDS data were obtained with a Leo field emission scanning electron microscope operated at $10-15 \mathrm{KV}$

Electrical Studies. The electrical measurements for determining the effect of hydrazine reduction on GO and GO-SFGN hybrids were taken at room temperature, under a steady nitrogen environment, using a Keithley 2612 dual-channel system source meter connected to a computer via a GPIB/IEEE-488 interface card. The temperature studies for determining the band gap and Schottky barrier height were carried out in a Janes cryostat, the temperature of which can be externally controlled by a Lake Shore 331 temperature controller.

Acknowledgment. We thank Heather Shinogle and Dr. David Moore for assistance with the FESEM and Dr. Dan Boyle for assistance with TEM imaging. We thank Dr. Qiang (Charles) Ye for his help with the Raman spectroscopic measurements. We also thank Nihar Mohanty for assistance with the electrical measurements. This work was supported by funds from Kansas State University (Start-up Grant).

Supporting Information Available: Description for synthesizing SFGN-coated GO sheets; their immobilization on silica substrates for FESEM, TEM, EDS, and SERS analysis, their electrical property measurements calculations for band gap and Schottky height measurements. This material is available free of charge via the Internet at http://pubs.acs.org.

\section{REFERENCES AND NOTES}

1. Novoselov, K. S.; Geim, A. K.; Morozov, S. V.; Jiang, D.; Zhang, Y.; Dubonos, S. V.; Grigorieva, I. V.; Firsov, A. A. Electric Field Effect in Atomically Thin Carbon Films. Science 2004, 306, 666-669.

2. Novoselov, K. S.; Geim, A. K.; Morozov, S. V.; Jiang, D.; Katsnelson, M. I.; Grigorieva, I. V.; Dubonos, S. V.; Firsov, A. A. Two-Dimensional Gas of Massless Dirac Fermions in Graphene. Nature 2005, 438, 197-200.

3. Lee, C.; Wei, X. D.; Kysar, J. W.; Hone, J. Measurement of the Elastic Properties and Intrinsic Strength of Monolayer Graphene. Science 2008, 321, 385-388.

4. Mohanty, N.; Berry, V. Graphene-Based Single-Bacterium Resolution Biodevice and DNA Transistor: Interfacing Graphene Derivatives with Nanoscale and Microscale Biocomponents. Nano Lett. 2008, 8, 4469-4476.

5. Geim, A. K.; Novoselov, K. S. The Rise of Graphene. Nat Mater. 2007, 6, 183-191.

6. Novoselov, K. S.; Jiang, D.; Schedin, F.; Booth, T. J.; Khotkevich, V. V.; Morozov, S. V.; Geim, A. K. TwoDimensional Atomic Crystals. Proc. Natl. Acad. Sci. U.S.A. 2005, 102, 10451-10453.

7. Sundaram, R. S.; Gomez-Navarro, C.; Balasubranianian, K.; Burghard, M.; Kern, K. Electrochemical Modification of Graphene. Adv. Mater. 2008, 20, 3050-3053.

8. Stankovich, S.; Dikin, D. A.; Piner, R. D.; Kohlhaas, K. A.; Kleinhammes, A.; Jia, Y.; Wu, Y.; Nguyen, S. T.; Ruoff, R. S. Synthesis of Graphene-Based Nanosheets via Chemical Reduction of Exfoliated Graphite Oxide. Carbon 2007, 45, 1558-1565.

9. Ryu, S.; Han, M. Y.; Maultzsch, J.; Heinz, T. F.; Kim, P.; Steigerwald, M. L.; Brus, L. E. Reversible Basal Plane Hydrogenation of Graphene. Nano Lett. 2008, 8, 4597-4602

10. Elias, D. C.; Nair, R. R.; Mohiuddin, T. M. G.; Morozov, S. V.; Blake, P.; Halsall, M. P.; Ferrari, A. C.; Boukhvalov, D. W.; Katsnelson, M. I.; Geim, A. K.; Novoselov, K. S. Control of Graphene's Properties by Reversible Hydrogenation: Evidence for Graphane. Science 2009, 323, 610-613.

11. Wehling, T. O.; Novoselov, K. S.; Morozov, S. V.; Vdovin, E. E.; Katsnelson, M. I.; Geim, A. K.; Lichtenstein, A. I.
Molecular Doping of Graphene. Nano Lett 2008, 8, 173-177.

12. Schedin, F.; Geim, A. K.; Morozov, S. V.; Hill, E. W.; Blake, P.; Katsnelson, M. I.; Novoselov, K. S. Detection of Individual Gas Molecules Adsorbed on Graphene. Nat. Mater. 2007, 6, 652-655.

13. Novoselov, K. S.; McCann, E.; Morozov, S. V.; Fal'ko, V. I.; Katsnelson, M. I.; Zeitler, U.; Jiang, D.; Schedin, F.; Geim, A. K. Unconventional Quantum Hall Effect and Berry'S Phase of $2 \pi$ in Bilayer Graphene. Nat. Phys. 2006, 2, 177180.

14. Novoselov, K. S.; Jiang, Z.; Zhang, Y.; Morozov, S. V.; Stormer, H. L.; Zeitler, U.; Maan, J. C.; Boebinger, G. S.; Kim, P.; Geim, A. K. Room-Temperature Quantum Hall Effect in Graphene. Science 2007, 315, 1379.

15. Stankovich, S.; Dikin, D. A.; Dommett, G. H. B.; Kohlhaas, K. M.; Zimney, E. J.; Stach, E. A.; Piner, R. D.; Nguyen, S. T.; Ruoff, R. S. Graphene-Based Composite Materials. Nature 2006, 442, 282-286.

16. Blake, P.; Brimicombe, P. D.; Nair, R. R.; Booth, T. J.; Jiang, D.; Schedin, F.; Ponomarenko, L. A.; Morozov, S. V.; Gleeson, H. F.; Hill, E. W.; Geim, A. K.; Novoselov, K. S. Graphene-Based Liquid Crystal Device. Nano Lett. 2008, 8, 1704-1708.

17. Bunch, J. S.; van der Zande, A. M.; Verbridge, S. S.; Frank, I. W.; Tanenbaum, D. M.; Parpia, J. M.; Craighead, H. G.; McEuen, P. L. Electromechanical Resonators from Graphene Sheets. Science 2007, 315, 490-493.

18. Abanin, D. A.; Novoselov, K. S.; Zeitler, U.; Lee, P. A.; Geim, A. K.; Levitov, L. S. Dissipative Quantum Hall Effect in Graphene near the Dirac Point. Phys. Rev. Lett. 2007, 98.

19. Hill, E. W.; Geim, A. K.; Novoselov, K.; Schedin, F.; Blake, P. Graphene Spin Valve Devices. IEEE Trans. Magn. 2006, 42, 2694-2696.

20. Zhang, Y. B.; Tan, Y. W.; Stormer, H. L.; Kim, P. Experimental Observation of the Quantum Hall Effect and Berry's Phase in Graphene. Nature 2005, 438, 201-204.

21. Witten, T. A.; Sander, L. M. Diffusion-Limited Aggregation. Phys. Rev. B 1983, 27, 5686-5697.

22. Williarris, G.; Seger, B.; Kamat, P. V. TiO 2 Graphene Nanocomposites. UV-Assisted Photocatalytic Reduction of Graphene Oxide. ACS Nano 2008, 2, 1487-1491.

23. Muszynski, R.; Seger, B.; Kamat, P. V. Decorating Graphene Sheets with Gold Nanoparticles. J. Phys. Chem. C 2008, 112, 5263-5266.

24. Si, Y. C.; Samulski, E. T. Exfoliated Graphene Separated by Platinum Nanoparticles. Chem. Mater. 2008, 20, 6792-6797.

25. Hummers, W. S.; Offeman, R. E. Preparation of Graphitic Oxide. J. Am. Chem. Soc. 1958, 80, 1339.

26. Gole, A.; Murphy, C. J. Seed-Mediated Synthesis of Gold Nanorods: Role of the Size and Nature of the Seed. Chem. Mater. 2004, 16, 3633-3640.

27. Gou, L. F.; Murphy, C. J. Fine Tuning the Shape of Gold Nanorods. Chem. Mater. 2005, 17, 3668-3672.

28. Jana, N. R.; Gearheart, L.; Murphy, C. J. Seeding Growth for Size Control of 5-40 nm Diameter Gold Nanoparticles. Langmuir 2001, 17, 6782-6786.

29. Murphy, C. J.; San, T. K.; Gole, A. M.; Orendorff, C. J.; Gao, J. X.; Gou, L.; Hunyadi, S. E.; Li, T. Anisotropic Metal Nanoparticles: Synthesis, Assembly, And Optical Applications. J. Phys. Chem. B 2005, 109, 13857-13870.

30. Ko, H.; Singamaneni, S.; Tsukruk, V. V. Nanostructured Surfaces and Assemblies as SERS Media. Small 2008, 4, 1576-1599.

31. Campion, A.; Ivanecky, J. E.; Child, C. M.; Foster, M. On the Mechanism of Chemical Enhancement in SurfaceEnhanced Raman-Scattering. J. Am. Chem. Soc. 1995, 117 11807-11808.

32. Tong, L. M.; Li, Z. P.; Zhu, T.; Xu, H. X.; Liu, Z. F. Single GoldNanoparticle-Enhanced Raman Scattering of Individual Single-Walled Carbon Nanotubes via Atomic Force Microscope Manipulation. J. Phys. Chem. C 2008, 112, 7119-7123. 
33. Nittmann, J.; Stanley, H. E. Tip Splitting Without Interfacial Tension and Dendritic Growth Patterns Arising from Molecular Anisotropy. Nature 1986, 321, 663-668.

34. Supriya, L.; Claus, R. O. Solution-Based Assembly of Conductive Gold Film on Flexible Polymer Substrates. Langmuir 2004, 20, 8870-8876.

35. Langer, J. S. Instabilities and Pattern-Formation in Crystal Growth. Rev. Mod. Phys. 1980, 52, 1-28.

36. Mullins, W. W.; Sekerka, R. F. Stability of Planar Interface During Solidification of Dilute Binary Alloy. J. Appl. Phys. 1964, 35, 444-\&.

37. Eda, G.; Fanchini, G.; Chhowalla, M. Large-Area Ultrathin Films of Reduced Graphene Oxide As a Transparent and Flexible Electronic Material. Nat Nanotechnol. 2008, 3, 270-274.

38. Lee, S. K.; Zetterling, C. M.; Ostling, M.; Aberg, I.; Magnusson, M. H.; Deppert, K.; Wernersson, L. E.; Samuelson, L.; Litwin, A. Reduction of the Schottky Barrier Height on Silicon Carbide Using Au Nanoparticles. Solid State Electron. 2002, 46, 1433-1440.

39. Gomez-Navarro, C.; Weitz, R. T.; Bittner, A. M.; Scolari, M.; Mews, A.; Burghard, M.; Kern, K. Electronic Transport Properties of Individual Chemically Reduced Graphene Oxide Sheets. Nano Lett. 2007, 7, 3499-3503. 